Check for updates

Cite this: Chem. Commun., 2018, 54,1738

Received 5th January 2018, Accepted 20th January 2018

DOI: $10.1039 / \mathrm{c} 8 \mathrm{cc} 00090 \mathrm{e}$

rsc.li/chemcomm

\section{Selective protein unfolding: a universal mechanism of action for the development of irreversible inhibitors $\uparrow$}

\author{
Samuel Askin, ${ }^{a}$ Thomas E. H. Bond, ${ }^{a}$ Alanna E. Sorenson, (D) a \\ Morgane J. J. Moreau, ${ }^{a}$ Helma Antony, ${ }^{a}$ Rohan A. Davis (DD $^{b}$ and \\ Patrick M. Schaeffer (D)*a
}

\begin{abstract}
High-throughput differential scanning fluorimetry of GFP-tagged proteins (HT-DSF-GTP) was applied for the identification of novel enzyme inhibitors acting by a mechanism termed: selective protein unfolding (SPU). Four different protein targets were interrogated with the same library to identify target-selective hits. Several hits selectively destabilized bacterial biotin protein ligase. Structureactivity relationship data confirmed a structure-dependent mechanism of protein unfolding. Simvastatin and altenusin were confirmed to irreversibly inactivate biotin protein ligase. The principle of SPU combined with HT-DSF-GTP affords an invaluable and innovative workflow for the identification of new inhibitors with potential applications as antimicrobials and other biocides.
\end{abstract}

Enzyme inhibitors are invaluable for the development of drugs, herbicides and pesticides. The potency and specificity of inhibitors are key to their successful applications in modern medicine and agriculture. Inhibitors can be either reversible or irreversible. Reversible inhibitors bind non-covalently to the target through a variety of mechanisms e.g. competitive, uncompetitive, noncompetitive and mixed inhibition. Irreversible inhibitors often react with the enzyme via covalent bond formation. In the last decades very few fundamentally new mechanisms of inhibition leading to inactivation of protein targets have been discovered. A new mechanism of action was recently unveiled for nucleozin, a potent inhibitor of the influenza nucleoprotein. ${ }^{1}$ Nucleozin aggregates nucleoprotein rendering its packaging in the virus impossible. ${ }^{2}$ Destabilizing compounds are commonly dismissed as they are assumed to be non-specific (e.g. chaotropic agents) but a few studies have shown that organometallic complexes ${ }^{3,4}$ and nanoparticles ${ }^{5}$ can selectively destabilize proteins. Other systems such as PROTAC can flag target proteins to the proteasome. ${ }^{6,7}$

\footnotetext{
${ }^{a}$ Centre for Biodiscovery \& Molecular Development of Therapeutics,

James Cook University, 142, James Cook Drive, Townsville, QLD 4811, Australia. E-mail: patrick.schaeffer@jcu.edu.au

${ }^{b}$ Griffith Institute for Drug Discovery, Griffith University, Brisbane, QLD 4111, Australia

† Electronic supplementary information (ESI) available: Experimental details and supplementary figures. See DOI: 10.1039/c8cc00090e
}

DSF and DSF-GTP are perfectly suited to screen compounds in high-throughput (HT) by measuring their effect on the thermal stability of a target protein. ${ }^{8-10}$

In this work we screened an identical library of 940 compounds with four different proteins in order to identify target selective hits (Fig. 1 and Fig. S1, ESI $\dagger$ ). We used HT-DSF-GTP to compare the effect of each compound on the thermal stability of $E$. coli (Ec) biotin protein ligase ${ }^{11}$ (BirA), dengue virus RNA-dependent RNA polymerase $^{9}$ (RdRp), the putative replicative DNA helicase (DnaB) and primase (DnaG) ${ }^{12}$ of Burkholderia pseudomallei (Bp).

All targets were produced as fusion proteins including both $\mathrm{N}$-terminal hexahistidine and C-terminal GFP tags as previously

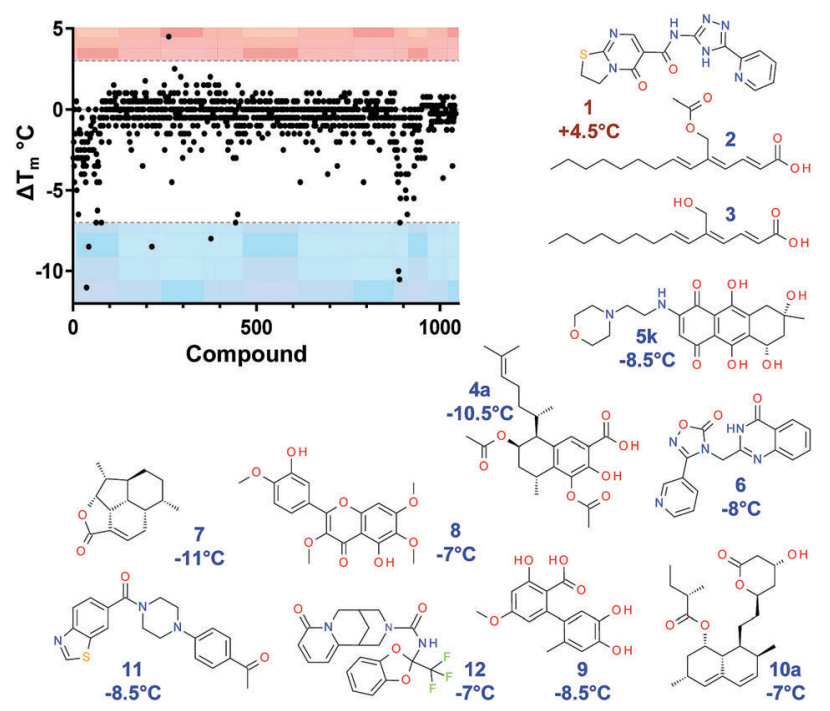

Fig. 1 HT DSF-GTP screening of 940 pure compounds with Ec BirA-GFP. Compounds ( $1 \mu \mathrm{L}$ at $5 \mathrm{mM}$ in DMSO, Compounds Australia) were dispensed in 96-well clear PCR plates (Biorad, HSP9601). Ec BirA-GFP (49 $\mu \mathrm{L}$ at $1 \mu \mathrm{M}$ ) was added to each compound for $15 \mathrm{~min}$ at RT prior subjecting the reactions to a melt-curve protocol $\left(30-85^{\circ} \mathrm{C}\right.$ at $\left.0.5{ }^{\circ} \mathrm{C} / 30 \mathrm{~s}\right)$ in a IQ5 iCycler (Bio-Rad). $T_{m}$ were analyzed as previously described. ${ }^{10}$ Structures of selected compounds and respective $\Delta T_{m}$ are shown. See ESI, $\dagger$ for detailed methods and Fig. S1 and S2A for extended data. 
described for RdRp-GFP, Bp DnaG-GFP, ${ }^{9}$ and BirA-GFP. ${ }^{11}$ Proteins $(1 \mu \mathrm{M})$ were reacted with compounds $(100 \mu \mathrm{M})$ in $96-$-well PCR plates for $15 \mathrm{~min}$ at room temperature before subjecting the reactions to a melt-curve protocol in a real-time PCR cycler (Fig. 1 and Fig. S1, ESI $\dagger$ ). Transition midpoint $\left(T_{\mathrm{m}}\right)$ values were recorded and processed as described previously using an automated $T_{\mathrm{m}}$ peak recognition software. ${ }^{10}$ For each compound, the net change in $T_{\mathrm{m}}\left(\Delta T_{\mathrm{m}}\right)$ was determined by subtracting the $T_{\mathrm{m}}$ of 'Target-GFP' from the $T_{\mathrm{m}}$ of 'Target-GFP + compound' in identical buffer conditions. After the initial screen (801 compounds from Open Collection (Scaffolds) and 139 from Open Access Natural Product-Based Library, Compounds Australia) it became evident that of the compounds that affected a protein, the majority decreased the proteins' $T_{\mathrm{m}}$. This is particularly obvious for Ec BirA-GFP (Fig. 1). From the initial screen, 94 hits were identified (i.e. the most stabilizing or destabilizing hits) and rescreened with all four targets to evaluate the reproducibility of the assay. Only four compounds that produced no distinguishable peak in the first screen were subsequently identified in the second screen.

The study was focussed on the hits identified in the Ec BirA-GFP screen as they had the largest effect on protein stability. A positive $\Delta T_{\mathrm{m}}$ cut-off value was set to $+3{ }^{\circ} \mathrm{C}$ which is equal to the increase in $T_{\mathrm{m}}$ of Ec BirA-GFP in the presence of $2 \mu \mathrm{M}$ of biotin. ${ }^{11}$

We also set a negative $\Delta T_{\mathrm{m}}$ cut-off value at $-7{ }^{\circ} \mathrm{C}$ due to the high number of destabilizing compounds identified for Ec BirA-GFP. Eleven selective hits were identified, with only one hit that significantly increased Ec BirA-GFP's $T_{\mathrm{m}}$ (Fig. 1 and 2A). The remaining 10 compounds thermally destabilized Ec BirA-GFP between -7 and $-11{ }^{\circ} \mathrm{C}$. These hits do not affect GFP (internal control) suggesting a target selective destabilizing mechanism. This is further supported by the fact that the remaining targets were mostly unaffected although in a different buffer. After rescreening and identification of false positives no compound destabilized GFP or any of the remaining protein targets $\geq 5{ }^{\circ} \mathrm{C}$ (see ESI, $\dagger$ Fig. S1).

The distribution of $\Delta T_{\mathrm{m}}$ values obtained for Ec BirA-GFP (Fig. 2A) shows that the majority of compounds does not significantly affect the $T_{\mathrm{m}}$ of Ec BirA-GFP (i.e. $\pm 2{ }^{\circ} \mathrm{C}$ ) with maximal effects recorded between -11 and $+4.5{ }^{\circ} \mathrm{C}$ (Fig. 2A). Only one stabilizing hit $1\left(\Delta T_{\mathrm{m}}=+4.5{ }^{\circ} \mathrm{C}\right)$ was identified with a $M_{\mathrm{W}}$ of 341 and $\log P$ value of 0.9 (Fig. 1 and 2B). No Ec BirA-GFP $T_{\mathrm{m}}$ peak was observed with structural analogues 2 and 3 (Fig. 1).
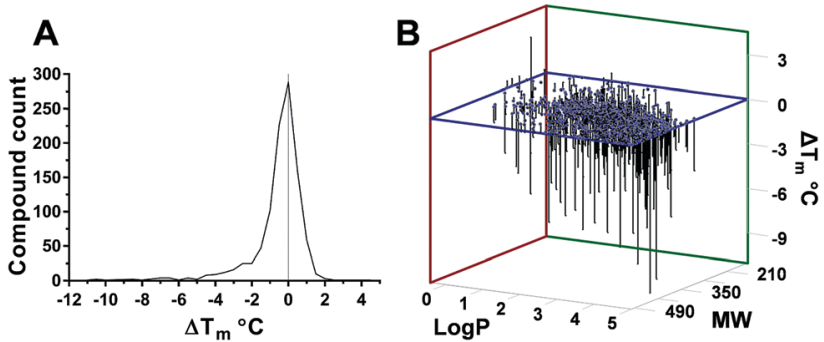

Fig. 2 (A) Statistical distribution of Ec BirA-GFP stabilizing and destabilizing compounds. (B) Correlation between $\log P$ and $M_{\mathrm{W}}$ of compounds, and their effect on Ec BirA-GFP $T_{m}$.
For destabilizing hits, a clear correlation can be seen between increasing $M_{\mathrm{W}}$ and $\log P$ values, and decreasing $T_{\mathrm{m}}$ (Fig. 2B). Destabilizing compounds are commonly written off as being non-specific. Strikingly, several of the Ec BirA selective destabilizing compounds were either serrulatanes ${ }^{13}$ 4a-e (Fig. 1 and Fig. S2B, ESI $\dagger$ ) or tetrahydroanthraquinones ${ }^{14,15}$ 5a-l (Fig. 1 and Fig. S2C, ESI $\dagger$ ) suggesting a structure-specific mechanism of protein destabilization. The strong structure-activity relationship (SAR) and striking correlation between the $\log P$ and $M_{\mathrm{W}}$ of Ec BirA selective destabilizing compounds lead us to hypothesize that they may present an untapped source of inhibitors.

First, we examined the concentration dependence of Ec BirA-GFP hits (see Fig. 3A and Fig. S3A, ESI $\dagger$ ), including stabilizing hit 1; destabilizing hits 2 and 3 which eliminated the $T_{\mathrm{m}}$ peak of Ec BirA, RdRp and Bp DnaG; serrulatanes $4 \mathbf{a}$ and 4b; 6; mitchellene $\mathrm{B}^{16} 7$, casticin 8, altenusin 9, lovastatin 10a, 11; and inactive compound Ia (see ESI, $\dagger$ Fig. S2A-D). All hits reduced the $T_{\mathrm{m}}$ of Ec BirA-GFP in a concentration-dependent fashion including 2 and 3 highlighting that for these the loss of $T_{\mathrm{m}}$ peak was due to protein unfolding at $100 \mu \mathrm{M}$.

Next, we examined SAR of four Ec BirA-GFP selective hits and four inactive compounds (see Fig. 3B and ESI, $\uparrow$ Fig. S3B for extended data). For each selected compound, we tested ten structural analogues on Ec BirA-GFP's $T_{\mathrm{m}}(\mathrm{V} 1-\mathrm{V} 10)$. No analogue of 1 and 6 affected Ec BirA-GFP but several analogues of both 11 and 12 significantly decreased its $T_{\mathrm{m}}\left(\Delta T_{\mathrm{m}}\right.$ range: -3.25 to $-6.75^{\circ} \mathrm{C}$; see ESI, $\dagger$ Fig. S2A and S3B). Finally, with the exception of two analogues of inactive compound Ib (see Fig. S2D and S3B, $\mathrm{ESI} \dagger)$ being slightly destabilizing $\left(\leq 2{ }^{\circ} \mathrm{C}\right)$, none of the others affected Ec BirA-GFP. The data supported that target selectivity of destabilizing hits is structure dependent.

Lovastatin 10a, a HMG CoA reductase inhibitor was identified as a Ec BirA-GFP destabilizing hit $\left(\Delta T_{\mathrm{m}}=-7{ }^{\circ} \mathrm{C}\right)$ in the initial screen. 10a was only little to moderately destabilizing towards DnaG-, DnaB- and RdRp-GFP (ESI, $\uparrow$ Fig. S1B). Simvastatin 10b (Fig. 4) has broad antimicrobial activity against various Grampositive pathogens and is also active against several Gramnegative pathogens e.g. Ec, Acinetobacter baumannii, Salmonella typhimurium, Klebsiella pneumoniae, and Pseudomonas aeruginosa with MIC ranging from $8-32 \mu \mathrm{g} \mathrm{mL}^{-1}$ when their outer
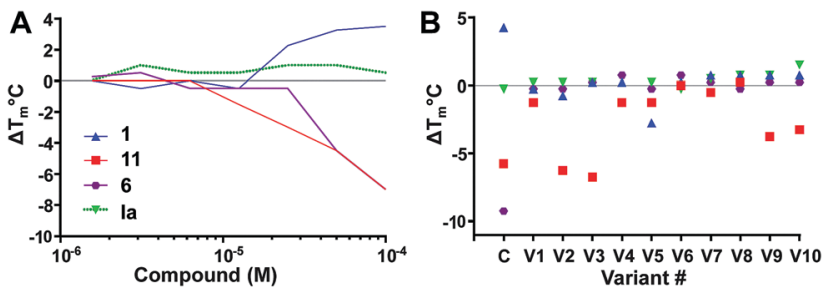

Fig. 3 Concentration dependence (A) and SAR studies (B). (A) Effect of two-fold serially diluted compounds on Ec BirA-GFP $T_{m}$. One inactive compound la (see ESI, $\dagger$ Fig. S2D) and eleven hits were selected from the initial screen. Only four compounds are shown for clarity (see ESI, $\dagger$ Fig. S3A for full data). (B) Effect of scaffold analogues on Ec BirA-GFP $T_{m}$. For each selected inactive compound and hits (Open Collection Scaffolds Library), ten analogues (V1-V10) were tested. The same four compounds are shown in panel A and B for clarity (see ESI, $\uparrow$ Fig. S3B for full data). 


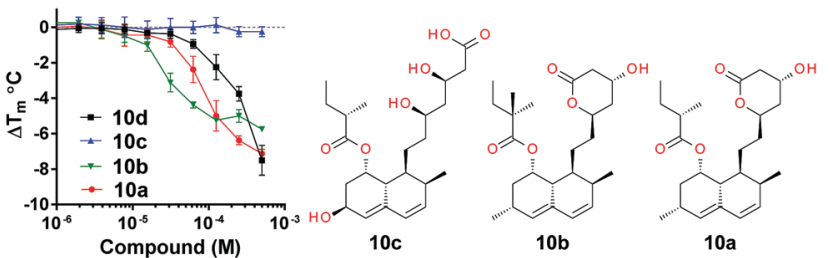

Fig. 4 SAR and concentration dependence of statins $10 \mathrm{a}-\mathrm{d}$ on $T_{\mathrm{m}}$ of Ec BirA-GFP. Error bars represent SD $(N=2)$. See ESI, $\uparrow$ Fig. S2A for structure of $10 \mathrm{~d}$.

membrane permeability is compromised using sub-inhibitory concentrations of colistin. ${ }^{17}$ This prompted us to perform a small scale statin-focussed SAR study. For this, 10a, 10b, pravastatin 10c and fluvastatin 10d were tested on Ec BirA-GFP (Fig. 4 and Fig. S2A, ESI $\dagger$ ). 10a, 10b and 10d destabilized Ec BirA-GFP, however 10b was more potent at lower concentrations and 10d was the least potent. 10c, an open lactone form, did not destabilize Ec BirA-GFP. This observation suggests an important role for the lactone ring of statins in BirA destabilization. It is important to note that a methyl to hydroxyl group substitution in 10c could also be the cause of its inactivity. With 10d, it is more difficult to draw any parallel conclusions as its structure is very different to the three other statins (see ESI, $\dagger$ Fig. S2A). Nevertheless we can hypothesize that the polyaromatic ring structure of 10d is most likely responsible for Ec BirA-GFP destabilization rather than its open lactone structure, and that its protein binding site is thus different to the site of binding of 10a and 10b.

Statins were further investigated with GFP-Basta ${ }^{18}$ in isothermal reactions to assess if they induce BirA aggregation (Fig. 5A). Ec BirA-GFP $(1 \mu \mathrm{M})$ was reacted with statins $(100 \mu \mathrm{M})$ in the presence of biotin, ATP and $\mathrm{MgCl}_{2}$ for $30 \mathrm{~min}$ at $37{ }^{\circ} \mathrm{C}$ before centrifugation and quantification of the soluble fluorescent protein fraction $\left(F_{\text {fold }}\right)$ by fluorimetry of the GFP domain (see ESI, $\uparrow$ for detailed methods). 10b was most destabilizing, leading to $\sim 70 \%$ protein aggregation after $10 \mathrm{~min}$ incubation at
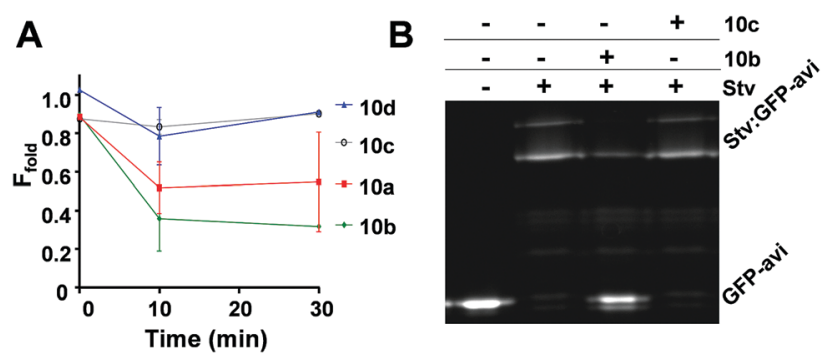

Fig. 5 Effect of statins on aggregation and biotinylation activity of Ec BirA-GFP. (A) Isothermal aggregation reactions ${ }^{18}$ were performed at $37^{\circ} \mathrm{C}$ with statins $10 \mathrm{a}-\mathbf{d}$ at $100 \mu \mathrm{M}$. Soluble fluorescent protein fraction $\left(F_{\text {fold }}\right)$ was determined by measuring residual fluorescence of Ec BirA-GFP in the reaction supernatant after centrifugation. Control reactions were performed without a statin. Error bars represent SD $(N=2)$. (B) Inhibition of Ec BirA-GFP biotinylation activity by $10 \mathrm{~b}$ and $10 \mathrm{c}$ at $37^{\circ} \mathrm{C}$. Inhibition of GFP-AviTag substrate biotinylation was determined by streptavidin (Stv)-induced band-shift and SDS-PAGE analysis. ${ }^{11}$ See Fig. 4 for structures of statins and $\mathrm{ESI}, \dagger$ for detailed methods.
$37{ }^{\circ} \mathrm{C}$ followed by 10a. Neither 10c nor 10d caused significant aggregation of Ec BirA-GFP over $30 \mathrm{~min}$ in these conditions. 10a and $\mathbf{1 0 b}$ were further tested on two orthologous BirA, i.e. Bp and Mycobacterium tuberculosis (Mt) BirA-GFP. ${ }^{19,20}$ These proteins share $\sim 30 \%$ sequence identity with each other and the Ec BirA. Both statins showed very little effect on Mt BirA-GFP at $100 \mu \mathrm{M}$ $\left(\Delta T_{\mathrm{m}}=-2{ }^{\circ} \mathrm{C}\right)$ and moderate destabilizing effects on Bp BirA-GFP $(c f$. ESI, $\dagger$ Fig. S4A) revealing a preferential destabilizing effect of 10a and 10b on Ec BirA. Using a GFP-based biotinylation activity assay, ${ }^{11,21}$ we confirmed that $10 \mathrm{~b}$ leads to Ec BirA-GFP inactivation (Fig. 5B). For this, Ec BirA-GFP was pre-incubated at $37^{\circ} \mathrm{C}$ with 10b or 10c for $1 \mathrm{~h}$ prior addition of the GFP-AviTag substrate and biotinylation reaction. Under these conditions, 10b inhibited Ec BirA-GFP activity by $83 \%$ (Fig. 5B, see ESI, $\dagger$ for detailed methods). As expected, 10c did not affect Ec BirA-GFP. Taken together, the data show that inhibition of biotinylation activity is associated with 10b-induced unfolding and aggregation of Ec BirA-GFP.

Altenusin 9 was identified as a potent and highly selective Ec BirA-GFP destabilizing hit $\left(\Delta T_{\mathrm{m}}=-8.5{ }^{\circ} \mathrm{C}\right)$. Indeed, 9 showed no effect on any other proteins (Fig. S1B, ESI $\dagger$ ). It has previously been shown to exert broad antimicrobial activity against several multidrug-resistant bacterial and fungal strains albeit not against Ec. ${ }^{22,23}$ Concentration dependence curves for Ec and the two orthologous Bp and Mt BirA-GFP showed that 9 was Ec selective. Only a small destabilizing effect was observed with Bp and Mt BirA-GFP in the presence of $100 \mu \mathrm{M}$ of 9 (Fig. 6A and Fig. S4B, ESI $\dagger$ ). The data clearly demonstrate that 9 inactivates Ec BirA-GFP at $25{ }^{\circ} \mathrm{C}$ (Fig. 6B, see ESI, $\dagger$ for detailed methods) through a highly selective protein unfolding (SPU) mechanism.

Target engagement in the cell ${ }^{24}$ as well as non-specific binding to serum proteins ${ }^{25}$ are major hurdles in drug development. We examined if 9, 10a and 10b were able to exert their destabilizing effect in a clarified bacterial cell lysate and in human serum (see ESI, $\dagger$ for detailed methods). DSF-GTP is the only DSF method that can selectively measure the $T_{\mathrm{m}}$ of a protein in the presence of other protein contaminants. DSF-GTP is thus perfectly suited for target engagement and serum interaction studies. All hits lost their ability to destabilize Ec BirA-GFP in the bacterial lysate even after heat treatment $\left(95{ }^{\circ} \mathrm{C}\right.$ for $\left.5 \mathrm{~min}\right)$ and clarification (see ESI, $\dagger$ Fig. S5). Since heat treatment denatures all but the most thermostable proteins, the loss of destabilizing activity could be due to a non-proteinaceous soluble factor in the bacterial lysate. In serum, significant differences in Ec BirA-GFP

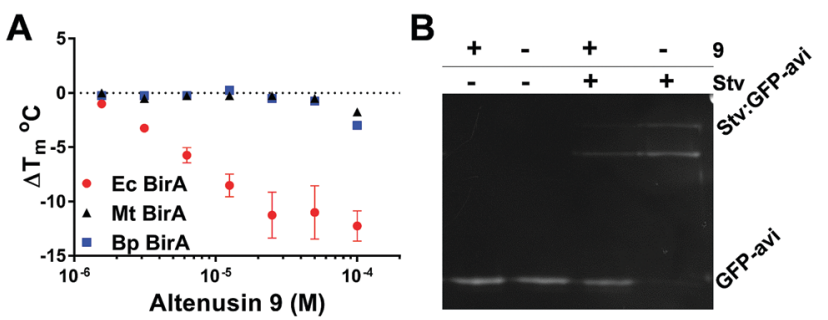

Fig. 6 (A) Concentration dependence of 9 on $T_{m}$ of Ec, Bp and Mt BirAGFP. Melt-curve protocol: $25-85^{\circ} \mathrm{C}$ at $0.5^{\circ} \mathrm{C} / 30$ s. Error bars represent SD $(N=2)$. (B) Inhibition of Ec BirA-GFP biotinylation activity by 9 at $25^{\circ} \mathrm{C}$ (see Fig. $5 \mathrm{~B}$ and $\mathrm{ESI}, \dagger$ for detailed methods). ${ }^{11}$ 
$T_{\mathrm{m}}$ were observed with the three different control reactions, with the heat-treated serum being the most destabilizing condition (see ESI, $\dagger$ Fig. S5). This suggest that one or more factors generated in the heat-treated serum are significantly affecting the $T_{\mathrm{m}}$ of Ec BirA-GFP. The heat-inducible destabilizing factor(s) can be partially eliminated after clarification of the heat-treated serum suggesting an aggregated proteinaceous factor. Most importantly and in stark contrast with the statins, 9 significantly destabilised Ec BirA-GFP in human serum. This effect is most pronounced in the heat treated and clarified serum (ESI, $\dagger$ Fig. S5).

10b has been shown to display a multitude of biological activities and clearly promiscuously binds several protein targets. In Staphylococcus aureus (SA), 10b significantly inhibits DNA, protein and lipid synthesis and to a lesser extent RNA synthesis and cell wall synthesis. ${ }^{17}$ BirA is the sole enzyme responsible for covalently attaching biotin at the active site of important metabolic enzymes, the biotin carboxylase and decarboxylases. ${ }^{26}$ The biotin carboxylases are essential enzymes in gluconeogenesis, lipogenesis, amino acid metabolism and energy transduction. Although the two orthologous $\mathrm{Mt}^{20}$ and $\mathrm{Bp}^{19}$ BirA tested in this study were not significantly destabilized by $\mathbf{1 0 b}$, it is important to note they are both type I enzymes whereas Ec BirA is a type II enzyme like Sa BirA. ${ }^{27}$ Thus, it is still tempting to propose BirA as a major target of $\mathbf{1 0 b}$ in susceptible bacteria whose inactivation would lead to a systematic inhibition of metabolic processes such as in $\mathrm{Sa}^{17}$ The fact that the bacterial lysate abolished the destabilizing activity of $\mathbf{1 0 b}$ on Ec BirA-GFP could indicate that it binds to further targets present in the bacteria or released after lysis. The high MIC value of $\mathbf{1 0 b}$ limits its use as a topical agent ${ }^{17}$ which is supported by our data in serum. Whether the antibacterial activity of $\mathbf{9}$ could also be improved against Gramnegative bacteria with the help of sub-inhibitory concentrations of colistin remains to be tested. Nevertheless, if entry of 9 into the bacteria can be achieved, and based on its activity in serum as well as its moderate cytotoxicity, it could become a promising lead.

Overall, our data clearly demonstrate that protein destabilizing compounds can be target- and species-selective (ESI, $\dagger$ Fig. S1B and Fig. 6A), and thus may offer quality lead compounds with applications in various fields of life sciences. Our data also highlight the unique application of HT DSF-GTP for screening protein-ligand interactions in complex matrices and identifying non-promiscuous hits. SPU as a mechanism of action combined with the power of HT DSF-GTP offers a fast and efficient platform for screening and identifying an exciting class of new inhibitors.

\section{Conflicts of interest}

There are no conflicts to declare.

\section{Notes and references}

1 H. Antony and P. M. Schaeffer, Analyst, 2013, 138, 6073-6080.

2 R. Y. Kao, D. Yang, L. S. Lau, W. H. Tsui, L. Hu, J. Dai, M. P. Chan, C. M. Chan, P. Wang and B. J. Zheng, et al., Nat. Biotechnol., 2010, 28, 600-605.

3 A. J. Wilson, K. Groves, R. K. Jain, H. S. Park and A. D. Hamilton, J. Am. Chem. Soc., 2003, 125, 4420-4421.

4 K. Groves, A. J. Wilson and A. D. Hamilton, J. Am. Chem. Soc., 2004, 126, 12833-12842.

5 N. O. Fischer, C. M. McIntosh, J. M. Simard and V. M. Rotello, Proc. Natl. Acad. Sci. U. S. A., 2002, 99, 5018-5023.

6 T. K. Neklesa, J. D. Winkler and C. M. Crews, Pharmacol. Ther., 2017, 174, 138-144.

7 K. M. Sakamoto, K. B. Kim, A. Kumagai, F. Mercurio, C. M. Crews and R. J. Deshaies, Proc. Natl. Acad. Sci. U. S. A., 2001, 98, 8554-8559.

8 M. W. Pantoliano, E. C. Petrella, J. D. Kwasnoski, V. S. Lobanov, J. Myslik, E. Graf, T. Carver, E. Asel, B. A. Springer and P. Lane, et al., J. Biomol. Screening, 2001, 6, 429-440.

9 M. J. J. Moreau, I. Morin, S. P. Askin, A. Cooper, N. J. Moreland, S. G. Vasudevan and P. M. Schaeffer, RSC Adv., 2012, 2, 11892-11900. 10 M. J. Moreau and P. M. Schaeffer, Mol. BioSyst., 2013, 9, 3146-3154.

11 S. P. Askin, T. E. H. Bond and P. M. Schaeffer, Anal. Methods, 2016, 8, 418-424.

12 P. M. Schaeffer, M. J. Headlam and N. E. Dixon, IUBMB Life, 2005, 57, 5-12. 13 E. C. Barnes, A. M. Kavanagh, S. Ramu, M. A. Blaskovich, M. A. Cooper and R. A. Davis, Phytochemistry, 2013, 93, 162-169.

14 V. Choomuenwai, K. T. Andrews and R. A. Davis, Bioorg. Med. Chem., 2012, 20, 7167-7174.

15 K. D. Beattie, N. Ellwood, R. Kumar, X. Z. Yang, P. C. Healy, V. Choomuenwai, R. J. Quinn, A. G. Elliott, J. X. Huang and J. L. Chitty, et al., Phytochemistry, 2016, 124, 79-85.

16 E. C. Barnes, A. R. Carroll and R. A. Davis, J. Nat. Prod., 2011, 74, 1888-1893.

17 S. Thangamani, H. Mohammad, M. F. Abushahba, M. I. Hamed, T. J. Sobreira, V. E. Hedrick, L. N. Paul and M. N. Seleem, Sci. Rep., $2015,5,16407$.

18 M. J. Moreau, I. Morin and P. M. Schaeffer, Mol. BioSyst., 2010, 6, 1285-1292.

19 T. E. H. Bond, A. E. Sorenson and P. M. Schaeffer, Microbiol. Res., 2017, 199, 40-48.

20 T. E. H. Bond, A. E. Sorenson and P. M. Schaeffer, Microbiol. Res., 2017, 205, 35-39.

21 A. E. Sorenson, S. P. Askin and P. M. Schaeffer, Anal. Methods, 2015, 7, 2087-2092.

22 J. Xiao, Q. Zhang, Y. Q. Gao, J. J. Tang, A. L. Zhang and J. M. Gao, J. Agric. Food Chem., 2014, 62, 3584-3590.

23 J. Kjer, V. Wray, R. Edrada-Ebel, R. Ebel, A. Pretsch, W. Lin and P. Proksch, J. Nat. Prod., 2009, 72, 2053-2057.

24 D. Martinez Molina, R. Jafari, M. Ignatushchenko, T. Seki, E. A. Larsson, C. Dan, L. Sreekumar, Y. Cao and P. Nordlund, Science, 2013, 341, 84-87.

25 M. Fasano, S. Curry, E. Terreno, M. Galliano, G. Fanali, P. Narciso, S. Notari and P. Ascenzi, IUBMB Life, 2005, 57, 787-796.

26 A. Chapman-Smith and J. E. Cronan, Jr., J. Nutr., 1999, 129, 477S-484S.

27 N. R. Pendini, M. Y. Yap, S. W. Polyak, N. P. Cowieson, A. Abell, G. W. Booker, J. C. Wallace, J. A. Wilce and M. C. Wilce, Protein Sci., 2013, 22, 762-773. 